\title{
MURRA EN LA SELVA DE PAJA ${ }^{1}$
}

\author{
Frank Salomon ${ }^{2}$
}

La última vez que vi a John Murra, en julio de 2006, estaba colgada frente a su cama una inmensa ampliación de una foto tomada en 1940 o 1941, en los pajonales de la sierra ecuatoriana. La ampliación fue regalo de su primer estudiante andinista estadounidense, Heather Lechtman, hoy profesora en el Massachusetts Institute of Technology. Esta imagen captó un primer momento de la vida andinista de Murra. En la foto, el joven Murra descansa montado en caballo, con las manos metidas en los bolsillos de una chaqueta de cuero. Están a su lado dos colegas ${ }^{1}$ y dos campesinos. La mirada de sus ojos negros expresa una atención fascinada, como si fuera el instante de observar por primera vez algo singular.

En su vejez el Dr. Murra solía mirar fijamente esta imagen. Durante minutos, posaba mentalmente dentro del remoto espacio de la foto. Cuando su atención volvía a los visitantes, preguntaba casi con ternura, ¿qué les pasó después a esos señores mis compañeros de viaje? Esta fase temprana y truncada de su carrera siguió siendo fuente subyacente de su inspiración mientras se desarrollaba como etnohistoriador. Este es el tema que quisiera subrayar en los párrafos que siguen.

En el quichua ecuatoriano el páramo alto, ventoso, frío, al parecer infinito, se llama ujsha sacha, o sea 'selva de paja'. Estos terrenos fueron los márgenes más remotos y amotinados de los dominios inkas. En el Ecuador los monumentos inkas son pocos y el daño que las guerras norteñas hicieron al Tawantinsuyu fue grave. Los pueblos de la 'selva de paja' contribuyeron directamente a la caída de los cusqueños en 1532-1535. Es de importancia el hecho de que Murra llegó a conocer el imperio Inka no por los portales de la ciudad sagrada del Cusco, sino atravesando estas tierras aguerridas y periféricas.

La excursión de Murra a la 'selva de paja' comenzó cuando leyó una nota en el tablero de anuncios diciendo que el Dr. Donald Collier del Field Museum buscaba un ayudante para el sitio ecuatoriano de Cerro Narrío, cerca de la frontera peruana. El joven Murra era en ese momento estudiante de postgrado en la Universidad de Chicago. Sus inquietudes se centraban en Africa, inspiradas por la docencia reveladora de A. R. Radcliffe-Brown. También le interesaban las tribus norteamericanas, cuya etnohistoria comenzaba a palpar estudiando las relaciones jesuíticas del imperio francés. No se interesaba en Suramérica ni sabía mucho de su historia. Sin embargo se ganó el puesto, porque en sus días como soldado en España había aprendido el español a la perfección.

Recién después de la muerte del Dr. Murra, recibí una carta de doña Costanza di Capua. Esta señora, residente de Quito fallecida en 2008, fue uno de los ecuatorianos que ayudaron a Murra en 1941. En aquel tiempo Costanza y su marido Alberto eran refugiados italo-judíos recién llegados a un país donde la ciencia apenas existía. Para ellos, como para muchos europeos varados en Suramérica debido a los desastres de la década de 1930, la antropología cosmopolita del joven rumano se conectaba con apetitos e inquietudes intelectuales que los arqueólogos norteamericanos poco comprendían. Doña Costanza se prestó como voluntaria para lavar y clasificar los restos obtenidos en Cerro Narrío. Ella opina que la primera semilla de la tesis de "verticalidad," formulada por el Dr. Murra en 1972, fue sembrada en 1941, cuando Collier y Murra se encontraron perplejos al descubrir entre los restos recién sacados del suelo del páramo alto, inmensas cantidades de concha marina Spondylus.

La expedición de Collier fue expulsada de la provincia de Loja cuando las fuerzas armadas peruanas cruzaron una frontera disputada y se produjo una guerra. Los antropólogos rápidamente inventaron un segundo proyecto ad hoc, pretendiendo poner a prueba la tesis de Max Uhle sobre una supuesta "marea" maya en la parte septentrional de Suramérica. Se dirigieron al norte del Ecuador, eventualmente a la provincia quichua-hablante de Imbabura. La investigación sobre la tesis de Uhle resultó poco estimulante para Murra. Pero desde el primer momento valoraba las excursiones a

Ensayo conmemorativo, École des Hautes Études en Science Sociale, Paris, 25 enero 2007.

John V. Murra Professor of Anthropology, University of Wisconsin, Wisconsin, EE.UU. fsalomon@wisc.edu 
caballo para reconocer sitios arqueológicos en las alturas. La foto aludida se tomó en una de estas salidas, en las alturas de Zula, cerca de Alausí en el centro-sur del país.

La precoz intervención de Murra en la antropología ecuatoriana correspondió a un momento cuando existía poquísima literatura sobre la población quichuahablante de los Andes septentrionales. Mientras Murra exploraba el sur del Ecuador, la antropóloga Elsie Clews Parsons (estudiante de Franz Boas) investigaba la cultura de los "indios mercaderes" de Otavalo, en el norte, entrevistando a la joven indígena Rosa Lema. La talentosa Lema nació en una familia de tejedores y mercaderes otavaleños y era empleada del famoso latifundista y estadista Galo Plaza Lasso. Parsons salió enferma del Ecuador en noviembre 1941, y murió poco después dejando inconcluso su estudio ${ }^{2}$. Al regresar a Chicago, Murra ayudó en la publicación de la obra póstuma de Parsons bajo el título de Peguche (University of Chicago Press, 1945, p.v), con la colaboración de Aníbal Buitrón. Peguche merece renovada atención no solamente por ser la primera monografía etnográfica moderna de los quichuahablantes ecuatorianos, sino también por ser uno de los primeros estudios cabalmente compilados desde el punto de vista femenino. En su madurez Mama Rosa Lema llegó a ser la primera "india ecuatoriana" en hablar ante la flamante Asamblea de las Naciones Unidas (1949) y en su larga vida llegó a ser renombrada matriarca de su sociedad. Murra, siempre interesado en los contactos entre indígenas e intelectuales, la mencionaba en sus cursos. En efecto, el contacto de Murra con los otavaleños en 1941, aunque fugaz, cundió en su mente. Proyectó un estudio detenido del fenómeno mercantil indígena otavaleño para su investigación doctoral, pero el proyecto quedó frustrado cuando el Departamento de Estado de EE.UU. le negó pasaporte.

La tesis de Magíster y las tres primeras obras de Murra fueron productos del viaje ecuatoriano. Entre éstas están sus dos capítulos en Handbook of South American Indians (1946, 1948). Todavía figuraban como las síntesis claves en 1970, cuando yo por primera vez toqué la puerta del departamento de Murra en Brooklyn. Por casualidad en mi etapa de estudiante y vagabundo me apasioné por los pueblos ecuatorianos, de manera muy parecida a lo que le pasó a Murra. Así principió la simpatía que guió nuestra relación académica.
El Dr. Murra no estimaba en mucho estas primeras publicaciones. Las describía como meros trabajos ocasionales para ganar algo. Recordando la experiencia de los años de 1940, en 1970-1974 Murra mostró ambivalencia sobre el manejo del Handbook por Steward. Al participar en el proyecto de etnografía comparada que Steward administraba en Puerto Rico, Murra se hizo amigo inseparable de Sidney Mintz ${ }^{3}$ y Eric Wolf. Los tres simpatizaron con el proyecto stewardiano de una antropología materialista, pero vieron el modelo "multilineal" de Steward como demasiado esquemático e insuficientemente historicista. Lamentaron la poca sensibilidad lingüística de Steward y su impaciente demanda por conclusiones "científicas". Murra vio a su amigo Alfred Métraux, cuyas labores conducentes al Handbook quedaron obstaculizadas por la Segunda Guerra Mundial, como el auténtico genio del proyecto, insuficientemente reconocido en la edición final. Pero a la vez que criticaba la tergiversación del Handbook Murra confesó admirar a Steward por su astucia al conseguir, dentro de la burocracia de Washington, las subvenciones necesarias para publicar obra tan inmensa, empaquetándola como una serie de informes anuales del Bureau of American Ethnology.

Desde el punto de vista de los estudiantes que aprendíamos antropología en la década de 1970, las actitudes del Dr. Murra a veces resultaban desconcertantes. No me refiero a sus famosos intervalos de mal humor, ni de su inaccesibilidad cuando sufría por insomnio o a causa de sus obscuras luchas psíquicas, ni de su vida privada que ocasionaba chismes, ni tampoco de su irritabilidad sobre el delicadísimo tema de su identidad judía anterior hasta asumir como nombre oficial su apodo y nom de guerre. Lo desconcertante fue su perfil de opositor singular dentro de la antropología norteamericana. No quiso tomar parte en ninguna de las tendencias que en ese tiempo rivalizaban para dominar la antropología, ni el marxismo, ni el estructuralismo, ni el determinismo ecológico, ni el modernismo desarrollista. La administración de la Universidad Cornell había contratado al Dr. Murra con la esperanza de poner "nueva locomotora" al programa de antropología aplicada que el recién fallecido Allan Holmberg había organizado en Vicos, Perú. Grande fue su sorpresa al descubrir que el Dr. Murra no simpatizaba con el desarrollismo protagonizado por los agrónomos y sociólogos. Sin embargo, el prestigio del Dr. Murra creció rápidamente en el campus, porque resultó 
muy adepto para organizar programas y conseguir fondos, dando al grupo latinoamericanista de Cornell un perfil más sofisticado.

Su postura de opositor nunca le impidió al Dr. Murra una viva participación en la comunidad académica. Le encantaba hablar y explicaba con elocuencia, con gracia, con ironía la historia de su marxismo juvenil, su desengaño, su vocación antropológica y sus esperanzas para la antropología sudamericana. Le resultó fácil encantar a la gente con su ingenio y su risa, su mucha cultura literaria y artística, sus cuentos picarescos y su generosidad. Su jardín fue escenario de alegría cuando nos venían a visitar investigadores extranjeros (entre ellos su querida amiga la lamentada colega Olivia Harris, bolivianista prematuramente fallecida en Londres en 2009). A pesar de discrepar con los movimientos teóricos de esa época, el Dr. Murra sabía dar la bienvenida a la gente de muchas tendencias, con la única condición, pero esa sí rígida, que tuviesen fuerte y sincera vocación etnográfica. Los "ismos" se dejaban en la puerta. Entrar en el seminario del Dr. Murra implicaba jurar un pacto tácito: etnografía (o etnohistoria) primero. Mientras el pacto se mantenía sólido, la atmósfera era cordial.

Cuando el Dr. Murra, junto con Nathan Wachtel y Jacques Revel compilaron el número especial de 1978 de Annales con el título de Anthropologie Historique des Sociétés Andines, ya había trabajado en Cornell durante 10 años y había enseñado regularmente en instituciones de EE.UU., Yale y Vassar College inclusive por 28 años. Había formado una generación de investigadores. Gracias en parte a los comentarios que Maurice Godelier venía publicando sobre su obra a partir de 1971, ya gozaba de una fama académica mucho más allá del ámbito latinoamericanista. Pero sólo en la larga perspectiva del momento actual resulta posible apreciar su gran impacto en el mundo de los estudiosos.

Un aspecto constante de su antropología es la mirada etnográfica desde la periferia del imperio antes que desde el centro. Al Dr. Murra le gustaba enseñar acerca de otro caminante de las periferias, el cronista Pedro Cieza de León, con quien sentía una afinidad especial. Cieza no era un letrado sino un soldado con un cuaderno. Cuando Cieza arribó a América era incluso más joven que Murra cuando llegó a España. Ya experimentado en las conquistas de las tierras que hoy conforman Colombia, en 1547, Cieza atravesó la misma 'selva de paja' ecuatorial en camino a las batallas que eventualmente someterían los conquistadores reacios a la corona. El cronista-soldado iba llenando sus alforjas con notas de campo extraordinarias, que escribía mientras el resto de la tropa dormía. Entrevistaba a los kurakas de múltiples etnias quienes se acordaban de los inkas como invasores. Así Cieza llegó a conocer el Tawantinsuyu al divagar por sus ásperos bordes septentrionales muy antes de escribir las famosas crónicas del Cusco, de la nobleza Inka y de los principios de la grandeza virreinal.

Algo similar fue el caso de Murra. Su visión del Tawantinsuyu como un Estado multiétnico, lleno de pueblos resistentes y heterogéneos, tuvo mucho que ver con el hecho de que la primera región del Tawantinsuyu que conoció, el Ecuador era en 1941, y todavía lo es, una sociedad mucho más fuertemente marcada por identidades étnicas definidas de lo que es el Perú. Murra llegó a poner sus ojos en el Cusco solamente en 1958, después de haber estudiado Ecuador y después de completar su estudio doctoral famoso sobre la economía política Inka. En 1960, cuando emprendió el proyecto arqueológico-etnohistórico de Huánuco Pampa (Perú) con el arqueólogo Craig Morris (también fallecido en 2006), el Dr. Murra ya se había comprometido principalmente con la etnohistoria de los pueblos andinos no-Inkas: lupaqas, chupaychus, yachas y yunkas. El lavado de cerebro garcilasiano que tanto cundió en la mentalidad euroamericana no le llegó a tocar. La visión de los dominios del imperio Inka como un sistema para articular la diversidad, antes que imponer la uniformidad, fue quizás su contribución más decisiva a los Annales de 1978 y la etnohistoria posterior.

Otra nota constante en la docencia del Dr. Murra fue el énfasis en el conflicto. Su percepción de ella como inevitable, por supuesto tuvo raíces autobiográficas. Era un niño de cuatro años en 1920 cuando sus padres lo sacaron de la ciudad de Odessa, entonces convulsionada por combates revolucionarios ${ }^{4}$. Como joven comunista rumano, peleaba con los fascistas del partido "Guardia Férrea," y estuvo preso varias veces. Sus padres le ayudaron a emigrar a los EE.UU., donde se alojó con un tío empleado, en ese momento, como contrabajista de un conjunto "gitano". Apenas egresado con su primer título de la Universidad de Chicago y recién casado, el joven inmigrante se inscribió como voluntario de la Brigada "Abraham Lincoln" para pelear en la guerra de España. Sufrió graves heridas durante la ofensiva del Ebro en 1938. Tras la 
derrota, su entonces esposa Virginia Miller lo rescató del campo de concentración en Francia. Regresado a los EE.UU. juzgó como imperdonable el abandono de los voluntarios republicanos expulsados, quienes sufrieron el descuido tanto de la URSS y sus simpatizantes como de sus países de origen. Muchos de ellos cayeron fatalmente en manos de los nazis. El rechazo de Murra al comunismo fue entonces temprano y tajante. Pero tampoco quiso acercarse a los anticomunistas; porfiaba como izquierdista independiente. Por consecuencia, cuando Murra pidió pasaporte, el Departamento de Estado erróneamente lo clasificó como sospechoso de simpatías soviéticas. Su lucha y litigio contra el maccartismo duró hasta 1956, año en el cual finalmente le fueron concedidos su pasaporte y su nueva ciudadanía. El costo de estos conflictos resultó inmenso. Entre la vuelta del Ecuador y el comienzo de su carrera peruana (1958) discurrieron 17 años conflictivos.

En un mundo saturado por las peleas, las obras de comprensión profunda y respetuosa, ya fuera novelística o etnográfica, figuraban en su pensamiento como victorias redentoras arduamente alcanzadas. $\mathrm{Su}$ antropología participaba de esta perspectiva. Los países, nos enseñaba, poseen antropologías -académicas o no, ilustradas o no- en virtud de su interconexión histórica con otros pueblos, incluso la interconexión mediante invasiones y guerras. Cieza de León fue parte integrante del desastre de la conquista, pero supo hacer algo humanamente admirable de esto. El resto de nosotros, también hijos de los imperios, no debemos considerarnos más inocentes que Cieza. La historia nos coloca al azar dentro del juego de muchos poderes peligrosos y nos toca hacer algo de bueno tomando esta situación como punto de partida. A los ojos del Dr. Murra no podría ni debería haber una ciencia universal de la antropología. Las antropologías se generan en las culturas y como subculturas de sus pueblos y tiempos particulares.

Característico del Dr. Murra también fue su creencia en la unidad de la antropología. No aceptaba la división de los estudios en "subdisciplinas" arqueológica, etnográfica, etc., sino denominaba a estas metodologías como "tácticas" dentro de una sola campaña. El eje de su complementariedad debía ser una visión historicista. Sin embargo, no faltaron desacuerdos sobre la manera de integrarlas. Ya en la temprana incursión ecuatoriana, y otra vez en la campaña de Huánuco Pampa, propuso utilizar las fuentes coloniales para poner prioridades: buscar la casa del kuraka mencionada por testigos tempranos, etc. Esta preferencia reflejaba su gran admiración por la obra de Moses Finlay (p.ej. The World of Odysseus 1954), pero iba en contra de la metodología propugnada por la arqueología de la post-guerra, dentro de la cual se proponía extraer del sitio arqueológico un máximo de datos materiales por métodos de muestreo extenso sin miras previas al contexto histórico.

John Murra se lanzó a candidato para presidente de la American Anthropologic Association en 1982. Su declaración de plataforma fue un manifiesto en contra de los tiempos. Se opuso a la inclusión de la antropología en las ciencias sociales. Argüía que la antropología americana debía reconocerse a sí misma específicamente como una consecuencia de las relaciones entre los pueblos europeos y los pueblos indígenas de América. Los proto-antropólogos como p.ej., John Wesley Powell y Garrick Mallery tuvieron antecedentes como militares enemigos de indios, pero esto no le parecía vergonzoso; ¿de dónde más podría venir el conocimiento etnográfico sino de los momentos conflictivos de la historia? Como un ejemplo de esa clase de etnografía históricamente ambivalente pero humanamente respetable, el Dr. Murra enseñaba acerca de Henry Rowe Schoolcraft. Schoolcraft fue geólogo y explorador estadounidense, varias veces comisionado por la entonces nueva república. A partir de 1822 se casó con una señora Ojibwe de Michigan e investigó entre los pueblos algonquinos y siouanos de la región septentrional de los Grandes Lagos. Fue implicado en un tratado desfavorable a la población originaria, pero también desarrolló una propuesta impecablemente respetuosa para instituir el estudio y reconocimiento de los pueblos nativos como obligación del Estado nacional. Un comentario del Dr. Murra sobre Lewis Henry Morgan en el curso History of US Anthropology ejemplifica su punto de vista: "Morgan no fue menos racista que los demás de su época. Pero avanzó más allá de su racismo".

La visión irreduciblemente pluralista e historicista del Dr. Murra fue una de las razones por las que él creía que los estudios andinos debían desarrollarse en instituciones andinas. Los Estados Unidos le ofrecieron una buena posición táctica porque después de 1957 el pánico producido por el Sputnik había abierto las compuertas por donde fluían los fondos dedicados a la investigación. 
Continuaba publicando mayormente en español y mayormente en el Perú, decisión no sin costos para su carrera académica. Fue uno de los miembros fundadores del Instituto de Estudios Peruanos y asiduo colaborador del Instituto Indigenista Interamericano en México. En las cartas que me escribió durante mi trabajo de campo, uno de sus constantes temas fue la lucha para financiar a estudiantes ciudadanos de países andinos y lo que fue aún más dificil, encontrar puestos decentes para ellos cuando regresaran a sus países de origen. Dedicó mucho tiempo y esfuerzo a los institutos y universidades en Chile, Perú, Argentina, España y México ${ }^{5}$. Se preocupaba de cómo establecer un adecuado intercambio de libros entre los países andinos, problema que hasta hoy no se ha resuelto. Durante un tiempo en los años 70 , el sótano de su casa se volvió una librería informal que despachaba, por correo, las visitas y otras obras importantes poco comerciadas. Otro de sus proyectos fue el de un boletín doble: Nispa Ninku ('Diciendo Dicen,' boletín de investigación) y Nispa Ninchis ('Diciendo Decimos,' boletín para diseminar la opinión andina acerca de la vida intelectual y política).

En 2000 sus alumnos chilenos Victoria Castro, Carlos Aldunate y Jorge Hidalgo resucitaron el título Nispa Ninchis y se lo dieron a la autobiografía conversacional de John Murra (Instituto de Estudios Peruanos e Instituto de Investigaciones Andinas, $2000)^{6}$. En el presente clima polarizado del discurso étnico andino vale la pena notar que Nispa Ninchis es una frase escrita en el "plural inclusivo primera persona" del quechua: esto es, significa "lo que nosotros, inclusive tú, decimos" más que "lo que nosotros, exclusive tú, decimos". Murra lamentaba que a pesar de su legendario multilingüismo nunca aprendió a conversar en quechua. De haberlo logrado, tal vez el título habría incluido el morfema de acción recíproca.

El Dr. Murra intencionó su obra como instrumentalidad para anular la maligna jerarquía que separaba los pueblos quechua y aymara-hablantes del poder y del diálogo académico. Su concepto de los reinos y kurakazgos "étnicos" y su proyecto para enseñar "historia andina" en las universidades suramericanas se relacionaron estrechamente con su percepción de la multi-etnicidad como el hecho central y artificialmente callado dentro de los países andinos. Para el Dr. Murra, el léxico de las clases sociales tanto como el de las razas redujo la idio- sincrasia del mundo andino a clichés convenientes para los dirigentes centralistas.

Murra con José María Arguedas se encontraban casi solos al tomar esta posición. En 1966, Arguedas dedicó al Dr. Murra su poema quechua "Huk dukturkunaman qayay" ("Llamado a unos doctores"). Escrito en víspera del primer intento de suicidio de Arguedas, el poema es grito de angustia e indignación contra ciertos científicos sociales quienes despreciaron su nueva novela Todas las Sangres por su contenido "indio". El último libro de John Murra (1996, con Mercedes López Baralt) explica el incidente caracterizándolo como momento importante en la historia intelectual. Los amigos chilenos de Murra se acuerdan de que Murra leyó este poema en el lanzamiento del libro Nispa Ninchis en Lima, abril de 2001. En un gesto dramático, abrió muy lentamente una carpeta y luego desdobló la copia original del diario de Lima donde se publicó el poema en comento. Antes de leerlo señaló que había sido en esta misma sala donde habían cuestionado a José María Arguedas, lo que en definitiva habría provocado la determinación de autoeliminarse. Cuando terminó de leer el poema el silencio fue total.

La amistad entre el Dr. Murra y Arguedas guarda cierto paralelismo con su entusiasmo por el "cronista indio" Felipe Guaman Poma de Ayala, otro genio bilingüe frustrado por la incomprensión de las élites frente a su reclamo étnico. Durante sus años en la Cornell, el Dr. Murra colaboró con la hispanista Rolena Adorno y el lingüista George Urioste para producir una edición anotada de la Nueva Corónica de Guaman Poma (1980 [1615]), hoy disponible en las páginas internet de la Biblioteca Real de Dinamarca. Este proyecto le dio enorme satisfacción, y con razón; un cuarto de siglo después sigue siendo recurso fundamental a los estudios andinos.

Las simpatías subjetivas tales como las que le conectaron con Arguedas y con Guaman Poma fueron para el Dr. Murra motivos respetables, que nunca debían ser obliterados a favor de las pálidas normas de la objetividad académica. Los profesionales que investigaban a tal o cual país con habilidad académica pero sin verdadera catexis afectiva no le provocaban admiración. En cambio nos enseñó a apreciar a los estudiosos distinguidos por su vocación andina, aun los sin mucha fama, como por ejemplo Toribio Mejía Xesspe, ayudante quechuahablante de Julio C. Tello e incansable amigo de caminar las punas. 
La gente frecuentemente le preguntaba al Dr. Murra cuáles asuntos teóricos lo impulsaron a escribir su estudio doctoral The Economic Organization of the Inca State. Él siempre respondió que la decisión no dependía en la teoría. Fue una respuesta a una experiencia temprana y decisiva. "No te olvides que había visto algo. Lo del Ecuador." Pero no era el Ecuador simplemente. “...En 1941-42 quedé deslumbrado por lo andino... allí vi todo lo que había, conocí la literatura y vi que era un problema muy bueno, muy grande... Yo estuve obligado a meterme a cosas andinas. Primero porque lo había visto; no era cosa de meras lecturas; lo había visto".

A pesar de sus opiniones minoritarias, el Dr. Murra llegó a ser el líder en importantes sectores de la antropología de los EE.UU. No era su programa lo que hacía a la gente congregarse a su alrededor en las reuniones y congresos. Era el placer que irradiaba al hablar de la disciplina, su inmensa erudición y su punto de vista siempre original que convencieron a los colegas. El Dr. Murra sirvió como presidente de la American Society for Ethnohistory (197071), la American Ethnological Society (1972-73), y el Institute for Andean Research (1977-83). En 1968 fue uno de cuatro delegados de EE.UU. en la conferencia de la Fundación Wenner-Gren donde se enfrentó la crisis ocasionada por el "Proyecto Camelot", una intervención clandestina de la CIA en la investigación antropológica. Dio las cuatro conferencias conmemorativas de Lewis Henry Morgan en la Universidad de Rochester en 1969, invitación considerada como uno de los más altos honores de la Antropología en EE.UU. Suficientes textos y grabaciones de estas conferencias inéditas se han recuperado para empezar un proceso editorial póstumo.

Hacia el final de sus días, toda lucha cesó y el combatiente anciano se volvió más suave. Al posar sus ojos en la foto suya en la 'selva de paja,' entendimos que al volver a la etnografía accidental de 1941 estaba saboreando un momento crucial de felicidad. ¿Qué había sido en aquel momento? ¿Un soldado de repente en paz? ¿Un observador principiante, que se daba cuenta que una vasta empresa de comprensión estaba a punto de abrirse? Es imposible decirlo. Pero hasta el final de su vida y a pesar de no haber renovado sus investigaciones en el norte andino, siguió guardando un cariño especial para los pueblos de la "selva de paja".

John V. Murra descansó en paz el 16 de octubre de 2006 en su casa en Ithaca, New York. Las comunidades antropológicas e históricas de su país de adopción, los Estados Unidos, como muchos otros, lamentan su fallecimiento. Sus papeles se conservan en el Archivo Antropológico Nacional de la Institución Smithsoniana en Washington.

\section{Notas}

1 Sus colegas más inmediatos durante la expedición ecuatoriana fueron Donald Collier y Aníbal Buitrón. No he podido encontrar los nombres de los campesinos.

2 Una monografía de David Kyle, con el título Transnational Peasants: Migrations, Networks, and Ethnicity in Andean Ecuador (Johns Hopkins University Press, 2000) narra partes de esta historia.
Ver Mintz 2010, en este número. Ver Harris 2010, en este número.

5 Ver Cereceda 2010, Hidalgo 2010, Silva 2010, en este número.

6 Ver Castro 2010, en este número. 